\title{
3D free-standing ordered graphene network geometrically regulates neuronal growth and network formation
}

Miao Xiao, 2, 6, Francesco Paolo Ulloa Severino ${ }^{4}$, Federico Iseppon 5 , Guosheng Cheng, ${ }^{6,}$, Vincent Torre ${ }^{2,3,}$, Mingliang Tang ${ }^{1, ~ *}$

${ }^{1}$ Institute for Cardiovascular Science \& Department of Cardiovascular Surgery of the First Affiliated Hospital, Medical College, Soochow University, Suzhou, 215000, China.

${ }^{2}$ Neurobiology Sector, International School for Advanced Studies (SISSA), via Bonomea 265, Trieste 34136, Italy.

${ }^{3}$ Laboratory of Translational Medicine, Suzhou Institute of Systems Medicine, Center for Systems Medicine, Chinese Academy of Medical Sciences, Suzhou Industrial Park, Jiangsu 215123, China.

${ }^{4}$ Cell Biology Department, Duke University Medical Center, 335 Nanaline Duke Building Duke University Medical Center, Durham, NC 27710, USA.

${ }^{5}$ Molecular Nociception Group, Wolfson Institute for Biomedical Research, University College London, Gower Street, London, WC1E 6BT.

${ }^{6}$ CAS Key Laboratory of Nano-Bio Interface, Suzhou Institute of Nano-tech and Nanobionics, Chinese Academy of Sciences, 398 Ruoshui Road, Suzhou Industrial Park, Jiangsu 215123, China. 


\section{Supplementary Figures}
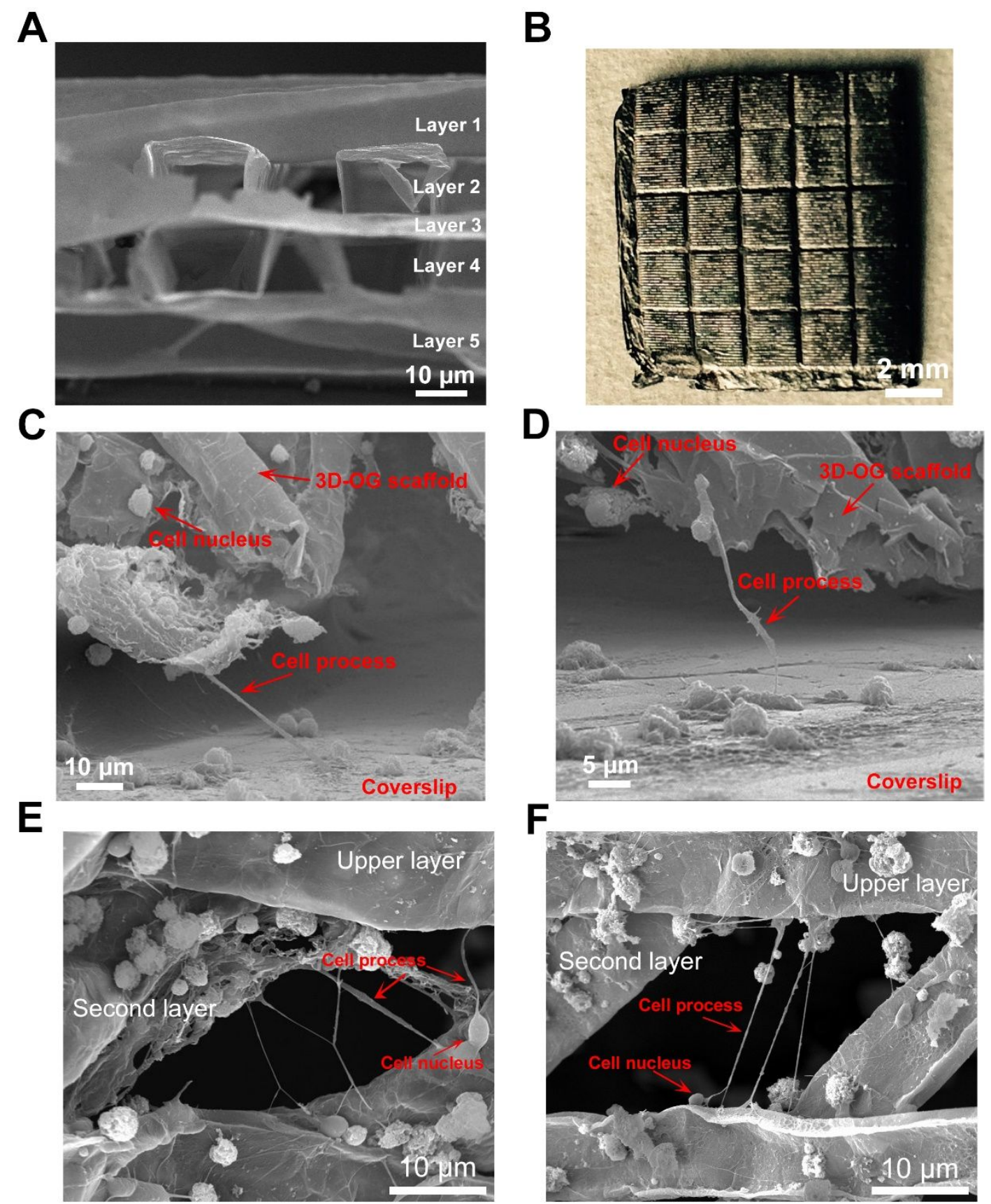

Supplementary Figure 1. Images to presented the 3D freestanding structure of 3D-OG scaffold and assembled with cortical cells after 8 days culture. A) SEM image showing the cross-section of the 3D-OG scaffold with 5 layers of graphene skeleton. B) Photograph of a typical macroscopic, freestanding 3D-OG scaffold. C and D) SEM images showing the cross-section view of the 3D-OG scaffolds assembled with cortical 
cells after 8 days culture. E and F) SEM images showing the top views of the 3D-OG scaffolds assembled with cortical cells after 8 days culture.
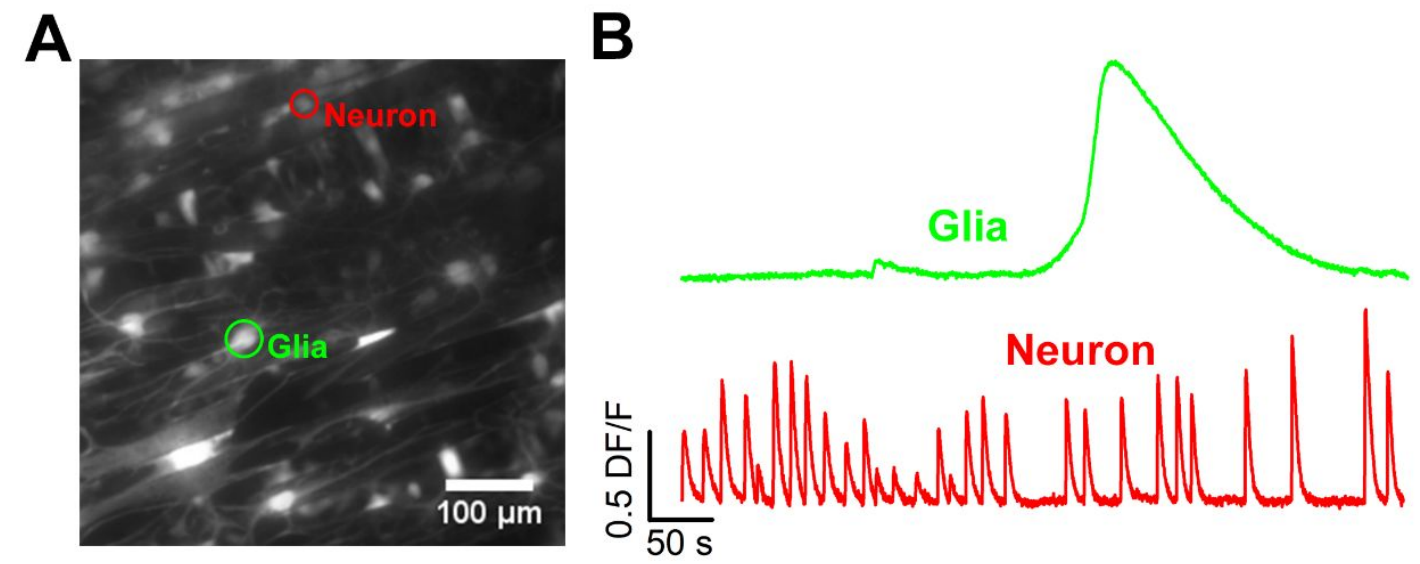

Supplementary Figure 2. Typical calcium transients from glial cells and neurons to identify the neurons from glial cells. A) Fluorescence image of a neuronal culture loaded with Fluo-4 AM grown on 3D-OG scaffold, also presented in Figure 4A. Red (green) circles indicate selected regions corresponding to the soma of neuron and glia cell. B) Optical transients from glial cells have a slow time course (upper portion of the panel) and those from neurons (lower portion, also presented in Figure 4B) have a faster rising phase.
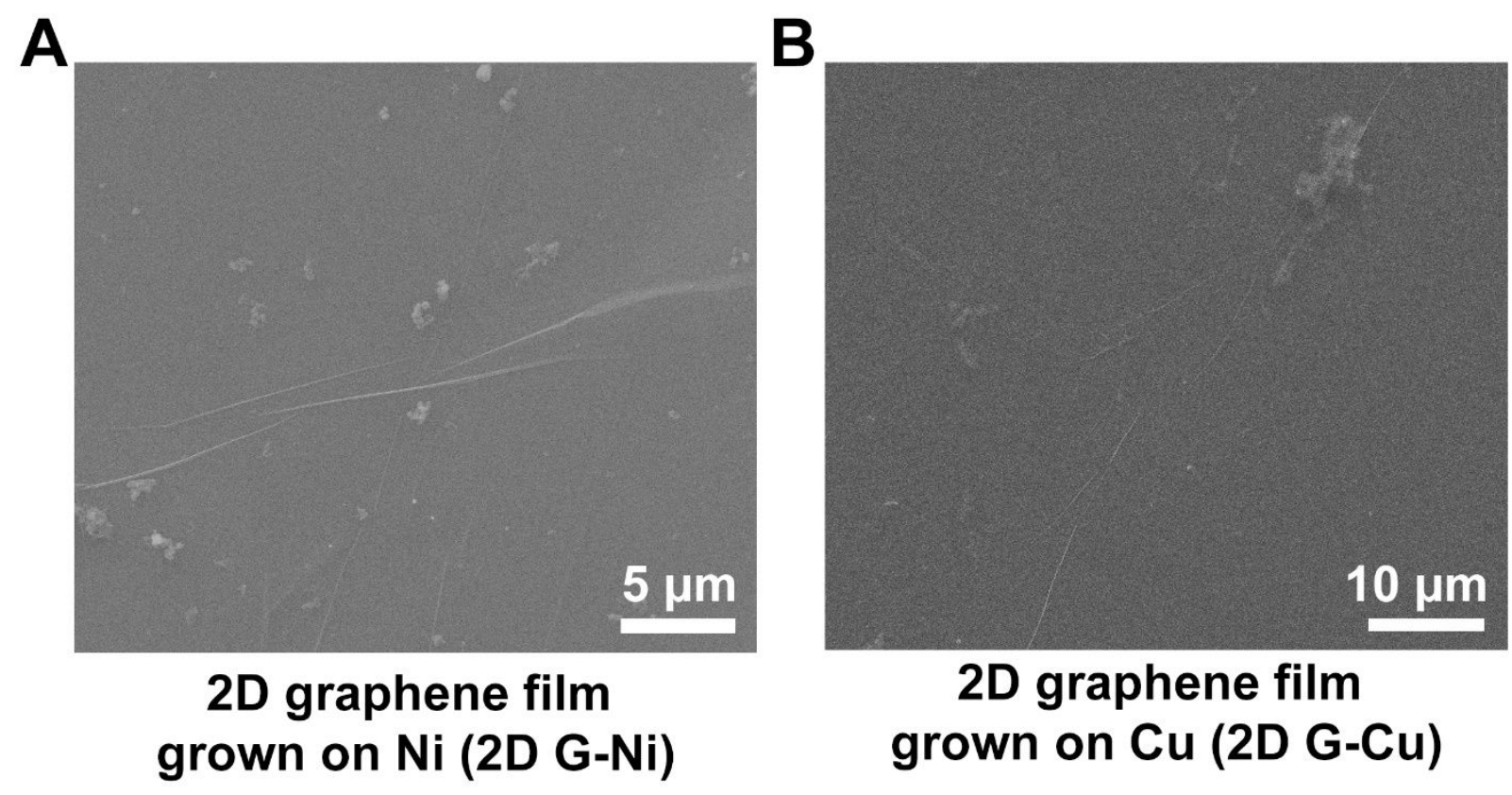
Supplementary Figure 3. The SEM images of 2D graphene film grown on Ni film (2D G-Ni) (A) and 2D graphene film grown on $\mathrm{Cu}$ film (2D G-Cu) (B).

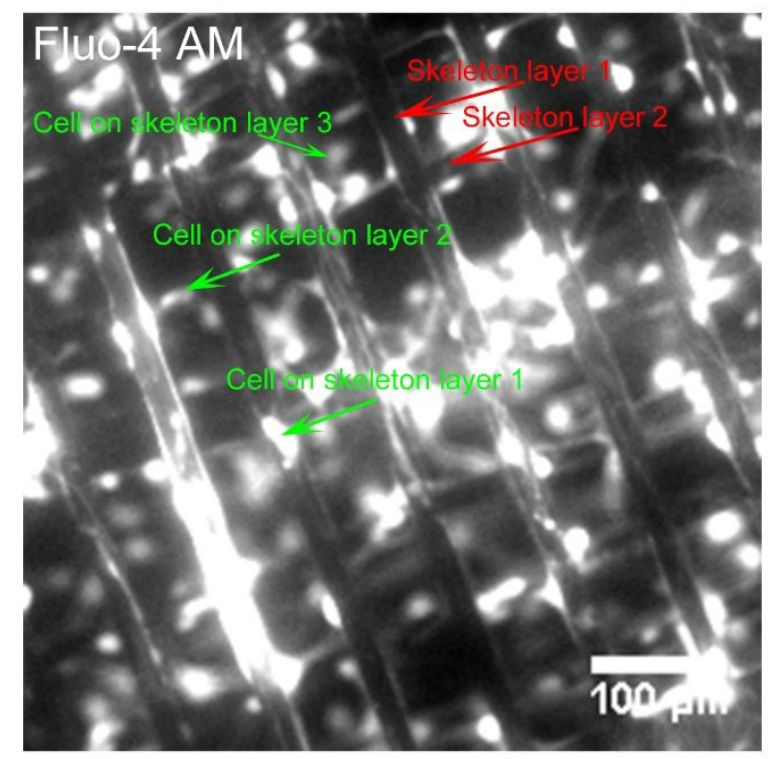

Supplementary Figure 4. The immune-fluorescence image of cortical cells loading with Fluo-4 AM on 3D-OG scaffolds (also presented in Figure 5B) to show the cells embedded in different skeleton layers.

\section{Supplementary Videos}

Supplementary Video 1. A Fluo-4 AM fluorescence calcium imaging of a cortical cell culture on 3D-OG scaffold for 8 days in vitro.

Supplementary Video 1. A converted color saturations of Fluo-4 AM fluorescence calcium imaging of a cortical cell culture on 3D-OG scaffold for 8 days in vitro.

\section{Experimental Section}

Fabrication processes of 3D free-standing ordered graphene network (3D-OG) and 2D graphene film.

The fabrication processes have been conducted as reported. ${ }^{17}$ Briefly, the ordered Ni templated with the pore size of $20 \mu \mathrm{m}$ and the skeleton width of $20 \mu \mathrm{m}$ were prepared following a typical top-down micro-fabrication strategy first, where a $150 \mathrm{~nm}$ thick $\mathrm{Au}$ 
film was deposited on $200 \mathrm{~nm}$ thick PMMA layer via magnetron sputtering (FHR Anlagenbau GmbH, Germany) as active electrode for electroplating. After desired pattern of AZ 4620 photoresist (AZ Electronic Materials, USA) was formed under photolithography and etching, the substrate was immersed into an electroplating bath for Ni deposition. The as-fabricated monolayer of Ni skeletons was then peeled off from the substrate and aligned with 5 monolayers. Afterwards, the Ni templates with specific angles were annealed at $700^{\circ} \mathrm{C}$ for $3 \mathrm{~min}$ in a muffle furnace (TM-0912P, China) to bond the monolayers together. The as-prepared 3D Ni scaffolds were placed into a chemical vapor deposition (CVD) system. The scaffolds were first annealed at $950^{\circ} \mathrm{C}$ for 10 min under $\mathrm{H}_{2}$ and $\mathrm{Ar}$ atmosphere to clean their surface and eliminate surface oxidation layers. Next, $\mathrm{H}_{2}$ and $\mathrm{CH}_{4}$ were introduced into the CVD system for $60 \mathrm{~min}$, followed by cooling down to room temperature to allow graphene growth on the $\mathrm{Ni}$ scaffold surfaces. The 3D-OG was finally obtained by sequentially rinsing with $1 \mathrm{M}$, $0.1 \mathrm{M}$ and $0.01 \mathrm{M} \mathrm{HCl}$ solutions, followed by rinsing with deionized water for 48 hours to remove residual etching agents.

The processes for graphene film grown on copper $(\mathrm{Cu})$ and nickel $(\mathrm{Ni})$ were similar to the 3D-OG scaffolds. Briefly, a thin $\mathrm{Cu}$ foil or a thin Ni film $(5 \mathrm{~cm} \times 5 \mathrm{~cm})$ was heated to $1000^{\circ} \mathrm{C}$ and annealed for 20 min under $\mathrm{H}_{2}$ and $\mathrm{Ar}$ atmosphere, followed by exposure to $\mathrm{H}_{2}$ and $\mathrm{CH}_{4}$ for $5 \mathrm{~min}$. The films were then cooled down from $1000^{\circ} \mathrm{C}$ to room temperature under $\mathrm{H}_{2}$ and Ar atmosphere. Graphene films were removed from the $\mathrm{Cu}$ foils or Ni films by etching in an aqueous solution of iron nitrate. Afterwards, glass coverslips were used to form contact with the graphene film which was pulled from the solution to produce the graphene film substrates. ${ }^{11}$

\section{Morphological and micro-structural characterizations of 3D free-standing ordered graphene network (3D-OG) and 2D graphene films.}

Field-emission scanning electron microscopy (SEM) equipped with energy dispersive spectrometer (EDS) (Quanta 400 FEG, FEI, USA), Raman spectrometer (LabRAMHR800, HORIBA, France) and transmission electron microscopy (TEM, 
Tecnai G2 F20 S-TWIN) were utilized to characterize the morphology and microstructure. For TEM observations, 3D-OG sheets were ultrasonically dispersed in ethanol for 45 min and then dropped onto a copper grid.

\section{Neuronal network preparation and culture.}

Cortical neurons from Wistar rats (P1-P3) were prepared in accordance with the guidelines of the Italian Animal Welfare Act, and their use was approved by the Local Veterinary Service, the SISSA Ethics Committee board and the National Ministry of Health (Permit Number: 630-III/14) in accordance with the European Union guidelines for animal care (d.1.116/92; 86/609/C.E.). To minimize the suffering of animals, the rats were anaesthetized with $\mathrm{CO}_{2}$ and sacrificed by decapitation quickly. During the culture process, OG scaffolds and all the other control substrates were first cleaned with $\mathrm{O}_{2}$ plasma and sterilized with ultraviolet (UV) rays. Then, they were coated with $50 \mu \mathrm{g}$ $\mathrm{mL}^{-1}$ poly-L-ornithine (Sigma-Aldrich, St. Louis, MO, USA) overnight, immersed in culture medium overnight and coated with Matrigel just before cell seeding (Corning, Tewksbury MA, USA). Dissociated cells were plated at a concentration of $2.4 \times 10^{6}$ cells $\mathrm{mL}^{-1}$ on 3D-OG scaffolds in a drop of minimum essential medium (MEM) with GlutaMAXTM supplemented with 10\% fetal bovine serum (FBS, all from Invitrogen, Life Technologies, Gaithersburg, MD, USA), 0.6\% D-glucose, $15 \mathrm{mM}$ Hepes, $0.1 \mathrm{mg}$ $\mathrm{ml}^{-1}$ apo-transferrin, $30 \mu \mathrm{g} \mathrm{ml}^{-1}$ insulin, $0.1 \mu \mathrm{g} \mathrm{ml}^{-1}$ D-biotin, $1 \mu \mathrm{M}$ vitamin B12 (all from Sigma-Aldrich) and $2.5 \mu \mathrm{g} \mathrm{ml}^{-1}$ gentamycin (Life Technologies). After 1 hour, Neurobasal supplemented with 2\% B-27, $10 \mathrm{mM}$ Glutamax (all from ThermoFisher) and $0.5 \mu \mathrm{M}$ Gentamycin (Sigma) was added as culture medium. Half of the medium was changed every week. Neuronal cultures were maintained in an incubator at $37^{\circ} \mathrm{C}$, $5 \% \mathrm{CO}_{2}$ and $95 \%$ relative humidity.

\section{Morphological and immunocytochemical analysis.}

Cells were fixed in 4\% paraformaldehyde in phosphate-buffered saline (PBS), saturated with $0.1 \mathrm{M}$ glycine, permeabilized with $0.1 \%$ Triton X-100, saturated with $0.5 \%$ BSA (all from Sigma-Aldrich) in PBS and then incubated overnight at $4{ }^{\circ} \mathrm{C}$ with primary 
antibodies: mouse monoclonal glial fibrillary acidic protein (GFAP) (Sigma-Aldrich) and anti- $\beta$-tubulin III (TUJ1) mouse monoclonal antibody (Covance, Berkeley, CA). The secondary antibodies were goat anti-mouse immunoglobulin (Ig) G1 Alexa Fluor ${ }^{\circledR}$ 488, goat anti-mouse IgG2a Alexa Fluor ${ }^{\circledR} 594$ (all from Life Technologies). The incubation time was 3 hours at room temperature $\left(20-22^{\circ} \mathrm{C}\right)$. Nuclei were stained with $2 \mu \mathrm{g} \mathrm{ml}^{-1}$ PBS Hoechst 33342 (Sigma-Aldrich) for 5 minutes. Samples were mounted in Vectashield (Vector Laboratories) on 1-mm thick coverslips with a homemade adaptor of PDMS to host the 3D samples. The cells were examined using a Nikon C2 confocal microscope to acquire high quality images. The fluorescence images were collected with a $20 \mathrm{X}$ magnification and $0.5 \mathrm{NA}$ objective. Each image was acquired with z-steps of $2 \mu \mathrm{m}$. Analysis of the image stack was accomplished using the NISElements AR software (Nikon), Volocity (PerkinElmer), and the open source imageprocessing package Fiji (http://fiji.sc/Fiji). During the process of calculating the curvature, initial shapes of neurites with spline curves were traced and then fitted to image data with a plugin, according to the instruction of Fiji. Then the data of every curve which indicated the neurite morphology and also the curvature values of each point from the curve were exported from Fiji. Neurite preferential alignments in networks were performed on the traces of fluorescent immunostaining images and the resultant polar plots were obtained using Neurolucida 360 (MBF Bioscience, VT, United States).

\section{Calcium imaging acquisition procedures.}

The cultured cells at 8 days in vitro (DIV 8) were loaded with a membrane-permeable calcium dye Fluo4-AM (Life Technologies) by incubating with $4 \mu \mathrm{M}$ Fluo4-AM [dissolved in anhydrous DMSO (Sigma-Aldrich), stock solution $4 \mathrm{mM}$ ] and Pluronic F-127 20\% solution in DMSO (Life Technologies) at a ratio of 1:1 in Ringer's solution (145 mM NaCl, $3 \mathrm{mM} \mathrm{KCl}, 1.5 \mathrm{mM} \mathrm{CaCl}_{2}, 1 \mathrm{mM} \mathrm{MgCl} 2,10 \mathrm{mM}$ glucose and $10 \mathrm{mM}$ Hepes, $\mathrm{pH} 7.4$ ) at $37^{\circ} \mathrm{C}$ for 1 hour. After incubation, the cultures were washed with Ringer's solution for 30 minutes and then transferred to the stage of a Nikon Eclipse Ti-U inverted microscope equipped with a piezoelectric table (Nano-ZI Series $500 \mu \mathrm{m}$ 
range, Mad City Labs), an HBO 103 W/2 mercury short arc lamp (Osram, Munich, Germany), a mirror unit (exciter filter BP 465-495 nm, dichroic $505 \mathrm{~nm}$, emission filter BP 515-555) and an Electron Multiplier CCD Camera C9100-13 (Hamamatsu Photonics, Japan). The calcium imaging recordings were performed at room temperature, and images were acquired using the NIS Element software (Nikon, Japan) with an S-Fluor $20 x / 0.75 \mathrm{NA}$ objective at a sampling rate of $3-5 \mathrm{~Hz}$ with a spatial resolution of $256 \times 256$ pixels for 10 minutes. To avoid saturation of the signals, excitation light intensity was attenuated by ND4 and ND8 neutral density filters (Nikon).

\section{Calcium imaging processing and analysis.}

The initial video was processed with the ImageJ (U. S. National Institutes of Health, Bethesda, MA) software. The image sequences were then analysed as previously described. Briefly, neurons were localized, and an appropriate region of interest (ROI) was selected to subtract the background. Appropriate ROIs around the cell bodies were then selected. The time course of the fluorescence intensity, $I_{f}(t)$, in this ROI was displayed, and any decay, which is a consequence of dye bleaching, was evaluated. The $\mathrm{Ca}^{2+}$ transients of each cell signal were extracted in a semi-automatic manner by selecting a threshold for the smallest detectable peak that was equal to three times the standard deviation of the baseline. Subsequently, the decay of $I_{\mathrm{f}}(\mathrm{t})$ was fitted to a cubic spline $[\mathrm{Y}(\mathrm{t})]$ interpolating $\mathrm{I}_{\mathrm{f}}(\mathrm{t})$ at 10 or 20 points. $\mathrm{Y}(\mathrm{t})$ was then added to the original optical signal to compensate for dye bleaching, and the fractional optical signal was calculated as follows: $\mathrm{DF} / \mathrm{F}=\left(\mathrm{Y}(\mathrm{t})+\mathrm{I}_{\mathrm{f}}(\mathrm{t})\right) / \mathrm{I}_{\mathrm{f}}(0)$, where $\mathrm{I}_{\mathrm{f}}(0)$ is the fluorescence intensity at the beginning of the recording.

\section{Computation of raster plot and correlation coefficient of calcium transient occurrence.}

The times, $t_{i}$, at which transient peaks occurred were presented in a conventional raster plot. To isolate the smaller transients from the larger ones, single traces were considered independently. The amplitude distribution of peaks was calculated to separate the two 
different classes of events. Based on this distribution, a threshold was set to approximately $30 \%$ of the maximum amplitude. All peaks under the threshold were considered small transients, whereas all other peaks were considered to be large calcium transients.

The correlation coefficient of the calcium transients for neuron $i$ and neuron $j\left(\sigma_{C T i j}\right)$ was computed as follows. The total recording time, $T_{\text {tot }}$, was divided into $N$ intervals $(1 . ., \mathrm{n}, \ldots, \mathrm{N})$ of a duration $\Delta t$. Thus, if $f_{\text {in }}$ and $f_{j n}$ are the numbers of calcium transients of neuron $i$ and neuron $j$ in the time interval $\Delta t_{n}$, then we have the following equation:

$\sigma_{C T_{i j}}=\frac{\sum_{n} f_{i n} f_{j n}}{\sqrt{\left(\sum_{n} f_{i n}^{2}\right)\left(\sum_{n} f_{j n}^{2}\right)}}$

where $\sigma_{C T i j}$ depends on $\Delta t$ and varies between 0 and 1 . The range of explored values of $\Delta t$ was $20 \mathrm{~s}$.

\section{Statistical analysis.}

Data were shown as mean \pm standard deviation (SD) from at least three neuronal culture preparations from different animals. The quantified activity (IEI and cross-correlation) and morphological data were analysed with the ANOVA test followed by post-hoc comparisons using the software Sygma Plot 10.0. Differences between two groups were evaluated with an unpaired t-test (Statistica 6.0-StatSoft Italy). Significance was set to $* \mathrm{p}<0.05, * * \mathrm{p}<0.01$ and $* * * \mathrm{p}<0.001$. 